Article

\title{
Plantago asiatica L. Ameliorates Puromycin Aminonucleoside-Induced Nephrotic Syndrome by Suppressing Inflammation and Apoptosis
}

\author{
Min Chul Kho ${ }^{1}$, Ji Hun Park ${ }^{1}$, Byung Hyuk Han ${ }^{1,2}$, Rui Tan ${ }^{1,2}$, Jung Joo Yoon ${ }^{1,2}$, \\ Hye Yoom Kim ${ }^{1,2}$, You Mee Ahn ${ }^{1,2}$, Yun Jung Lee ${ }^{1,2}$, Dae Gill Kang 1,2,* and Ho Sub Lee 1,2,* \\ 1 Hanbang Body-fluid Research Center, Wonkwang University, 460 Iksandae-ro, Iksan, Jeonbuk 54538, Korea; \\ shadowzetx@hanmail.net (M.C.K.); jihuncjstk@naver.com (J.H.P.); arum0924@nate.com (B.H.H.); \\ tanrui@hanmail.net (R.T.); morality16@hanmail.net (J.J.Y.); hyeyoomc@naver.com (H.Y.K.); \\ aum2668@naver.com (Y.M.A.); shrons@wku.ac.kr (Y.J.L.) \\ 2 College of Oriental Medicine and Professional Graduate School of Oriental Medicine, Wonkwang University, \\ 460 Iksandae-ro, Iksan, Jeonbuk 54538, Korea \\ * Correspondence: dgkang@wku.ac.kr (D.G.K.); host@wku.ac.kr (H.S.L.); Tel.: +82-63-850-6447 (H.S.L.)
}

Received: 18 November 2016; Accepted: 6 April 2017; Published: 14 April 2017

\begin{abstract}
Objective: Nephrotic syndrome, a kidney disease with a variety of causes, is mainly characterized by heavy proteinuria, hypoproteinemia, and ascites. This study was designed to evaluate the underlying mechanism of action of Plantago asiatica L. (PAL) in treating nephrotic syndrome induced by puromycin aminonucleoside. Methods: PAL has been used in Asia as a traditional medicine and dietary health supplement. Sprague-Dawley (SD) rats were intravenously injected with puromycin aminonucleoside $(75 \mathrm{mg} / \mathrm{kg} /$ day), then treated with either Losartan ( $30 \mathrm{mg} / \mathrm{kg} /$ day) or PAL ( $200 \mathrm{mg} / \mathrm{kg} /$ day) by oral gavage for seven days. Results: PAL significantly decreased ascites, proteinuria level, and plasma lipid parameters. In addition, treatment with PAL attenuated histological damage and hypoalbuminemia. Treatment with PAL also restored podocin expression and reduced inflammation markers such as intracellular adhesion molecules (ICAM-1), monocyte chemotactic protein-1 (MCP-1), tumor necrosis factor alpha (TNF- $\alpha$ ) and high-mobility group box-1 (HMGB1). Lower expression levels of the apoptosis markers Bax, caspase-3 and capase-9 were documented in SD rats receiving PAL. PAL also significantly decreased the phosphorylation levels of MAPKs such as ERK, JNK and p38. Conclusion: As a multifunctional agent, PAL has a renoprotective effect in nephrotic syndrome rat models. The anti-inflammatory and anti-apoptotic properties, along with reductions in hyperlipidemia and ascites, represent important therapeutic effects. These results indicate that Plantago asiatica is likely to be a promising agent in the treatment of nephrotic syndrome.
\end{abstract}

Keywords: nephrotic syndrome; plantago asiatica; inflammation; apoptosis; ascites

\section{Introduction}

Nephrotic syndrome (NS), a kidney disease with a variety of causes, is mainly characterized by heavy proteinuria, hypoproteinemia and ascites [1]. NS patients suffer from severe complications such as hypercoagulation, thrombotic symptoms, and disorders of metabolism [2].

The pathogenesis of NS is complex and not entirely clear, involving the circulatory and metabolic systems among others. Many studies suggest that renal inflammation, oxidative stress and apoptosis are typically associated with the development and progression of NS pathological features [3-5]. Various factors associated with the progression of glomerulonephritis, nephrotoxicity, and other renal disorders, such as chemokines and adhesion molecules, are secreted by resident renal cells. 
These factors attract and induce infiltration by circulating inflammatory cells [6]. In addition, pro-inflammatory cytokines increase synthesis of other inflammatory molecules and trigger cell apoptosis [7]. Apoptosis caused by environmental and intrinsic stimuli results in a loss of resident kidney cells, and subsequent development of kidney injury [8]. Therefore, in proteinuric renal disorders, such as nephrotic syndrome and chronic renal disease, inflammation and apoptosis are closely linked to, and implicated in, renal injury [9].

Traditional herbal medicines have been used, with apparent safety and efficacy, to treat and alleviate various complex refractory diseases, such as cancer and nephrotic syndrome [10-13]. Plantago asiatica L. (PAL) has been used for more than 2000 years in Asia as a traditional medicine and dietary health supplement [14]. In addition, analysis certification of PAL was approved by permission of the Korea food and drug administration (KFDA, PLAS2014). According to this certificate of analysis, Geniposidic acid and acteoside are main ingredients of PAL. It has previously been reported that extract of PAL was found to have anti-depressant, hypoglycemic, anti-oxidant, and lipid metabolism regulating effects [15-17]. However, renoprotective effects of PAL have not been reported thus far. Thus, the aim of the present study was to investigate the renoprotective effects of PAL in nephrotic syndrome in terms of renal function, inflammation, and apoptosis.

\section{Materials and Methods}

\subsection{Preparation of Seed of Plantago asiatica $L$.}

Dried raw seed of Plantago asiatica L. (PAL) herb was purchased from the Herbal Medicine Co-operative Association, Iksan, Jeonbuk Province, Korea. PAL seed (200 g) was soaked in double-distilled water, then boiled with $1.5 \mathrm{~L}$ of distilled water at $100^{\circ} \mathrm{C}$ for $2 \mathrm{~h}$. The extract was filtered through Whatman No. 3 filter paper (Whatman International Ltd., Maidstone, UK) and centrifuged at $990 \times g$ for $20 \mathrm{~min}$ at $4{ }^{\circ} \mathrm{C}$. Supernatant was concentrated using a rotary evaporator and the resulting extract (13.12 g) was lyophilized using a freeze-drier. The extract voucher specimen (No. HBJ101) was kept at $4{ }^{\circ} \mathrm{C}$ and deposited in the herbarium of the Professional Graduate School of Oriental Medicine, Wonkwang University (Iksan, Jeonbuk, Korea). The extract was dissolved in distilled water before use.

\subsection{Drugs and Chemicals}

The puromycin aminonucleoside for injection was purchased from Enzo Life Sciences, Inc. (Farmingdale, NY, USA). Losartan, used as the positive control drug, was purchased from Santa Cruz Biotechnology (Santa Cruz, CA, USA).

\subsection{Animal Experiments and Treatment Protocol}

All experimental protocols involving the use of animals were conducted in accordance with National Institutes of Health (NIH) guidelines and approved by the Institutional Animal Care and Utilization Committee for Medical Science of Wonkwang University (WKU12-12). Eight-week-old male Sprague-Dawley (SD) rats (weighing 170-200 g) were obtained from Samtako (Osan, Korea). Rats were kept in a room with automatic maintenance of temperature $\left(23 \pm 2{ }^{\circ} \mathrm{C}\right)$, humidity $(\sim 50 \%-60 \%)$, and 12-h light/dark cycle throughout the experiments. During the experiments, all animals were given standard rat chow diet ad libitum. Rats were randomly divided into four groups: Control group; a puromycin aminonucleoside-treated NS group; a NS plus Losartan (30 mg/ $\mathrm{kg} /$ day) treated group; and a NS plus PAL ( $200 \mathrm{mg} / \mathrm{kg} /$ day) treated group. Rats were intravenously injected with puromycin aminonucleoside ( $75 \mathrm{mg} / \mathrm{kg} /$ day, dissolved in saline). The control group was injected with saline only. Rats were administered either distilled water (Control and NS groups), Losartan or PAL by an oral gavage method once daily for 7 days. During the experiments, all animals were housed separately in a metabolic cage and 24 -h urine samples were collected before sacrifice. On day 7 , the end of the study period, all rats were sacrificed for blood and renal tissues. Blood samples were collected and centrifuged to separate plasma at $600 \times \mathrm{g}$ for $15 \mathrm{~min}$ at $4{ }^{\circ} \mathrm{C}$. The weight of the ascitic 
fluid was measured by inserting a weighed tissue paper into the abdomen to absorb the fluid before reweighing it.

\subsection{Measurement of Urinary Protein Excretion}

For urinary protein analysis, each animal was housed separately in a metabolic cage and 24-h urine samples were collected after puromycin aminonucleoside injection. Urinary protein concentrations were determined using Bradford Dye Reagent (Bio-Rad, München, Germany).

\subsection{Measurement of Plasma Biochemical Parameters}

Plasma total cholesterol (T-Cho), triglyceride (TG), low-density lipoprotein cholesterol (LDL-c), blood urea nitrogen (BUN), total protein (T-pro) and albumin (Alb) levels were measured using commercially available kits (ARKRAY, Inc., Minami-ku, Kyoto, Japan). Plasma creatinine was measured by a colorimetric method using a spectrophotometer [18] (Miloton Roy, Rochester, NY, USA). Pre-chilled EDTA-coated tube was used as an anticoagulant (BD Vacutainer, BD Franklin Lakes, NJ, USA).

\subsection{Histopathological Staining of Renal Tissues}

For morphological staining, isolated kidney tissues were fixed with $10 \%$ formalin, dehydrated in graded alcohol, and embedded in paraffin. Sections measuring $5 \mu \mathrm{m}$ were cut and stained with periodic acid-Schiff (PAS) reagent. All slides were evaluated by the same pathologist, who was unaware of the nature of the experimental groups. Images of kidney sections were taken with Axiovision 4 Imaging/Archiving software. For quantitative analysis, the average score of 10-20 randomly selected areas was calculated using image analysis software. Images were analyzed for protein cast formation using the Image J (NIH, Bethesda, MD, USA) program to select and quantify PAS-stained areas as a percentage of the total area of each image.

\subsection{Immunohistochemical Staining of Renal Tissues}

Kidney samples were fixed with $10 \%$ formalin and embedded in paraffin. After antigen retrieval to suppress endogenous peroxidase activity, slides were immersed in $3 \%$ hydrogen peroxide for $10 \mathrm{~min}$. Sections were incubated with the appropriate primary in humidified chambers overnight at $4{ }^{\circ} \mathrm{C}$. Viewing was performed using the $3,3^{\prime}$-diaminobenzidine (DAB; Novex ${ }^{\circledR}$, San Diego, CA, USA) substrate-chromogen system, with hematoxylin (Zymed, San Francisco, CA, USA) counterstaining. Image J software was used for quantitative analysis.

\subsection{Western Blot Analysis}

Renal tissue homogenates ( $40 \mu \mathrm{g}$ of protein) were separated using 10\% SDS-polyacrylamide gel electrophoresis and transferred to nitrocellulose paper membranes. Blots were then blocked by $5 \%$ BSA powder in Tris-buffered saline (TBS) for $1 \mathrm{~h}$, and incubated with appropriate primary antibodies to Podocin, ICAM-1, MCP-1, HMGB-1, TNF- $\alpha$, Bcl-2, Bax, Caspase-3, Caspase-9, phospho-p38, p38, phospho-ERK1/2, ERK1/2, phosphor-JNK, JNK or $\beta$-Actin (Santa Cruz Biotechnology, Santa Cruz, CA, USA). Subsequently, the membrane was incubated with a secondary goat anti-rabbit IgG or goat anti-mouse IgG antibody conjugated to horseradish peroxidase (Enzo Life Sciences, Farmingdale, NY, USA). The bands were visualized with enhanced chemiluminescence (Amersham, Buckinghamshire, UK). Protein expression levels were determined by analyzing signals captured on the nitrocellulose membranes using the ChemiDoc image analyzer (Bio-Rad, Hercules, CA, USA).

\subsection{Statistical Analysis}

Results were expressed as mean $\pm \mathrm{SE}$, and the data were analyzed using one-way ANOVA followed by student's $t$-test using SIGMAPLOT (Sigma plot ver. 10.0) to determine any significant differences. Significance was set at $p<0.05$. 


\section{Results}

\subsection{Effects of PAL on Proteinuria and Ascites}

Proteinuria is an important characteristic of puromycin aminonucleoside-induced NS in rats. As shown in Figure 1A, 24-h urinary protein excretion progressively increased following puromycin aminonucleoside injection. Proteinuria levels in the NS group peaked on day 7 compared to the Control group. However, the PAL-treated group exhibited significantly decreased proteinuria levels on day $7(p<0.01)$. Similarly, treatment with losartan produced similar results to those seen in the PAL group. Ascites is another important characteristic of puromycin aminonucleoside-induced rat NS. As shown in Figure 1B, the NS group had significantly increased ascites volume compared with the Control group. Both the PAL- and Losartan-treated groups had significantly decreased ascites volume.

$\mathbf{A}$

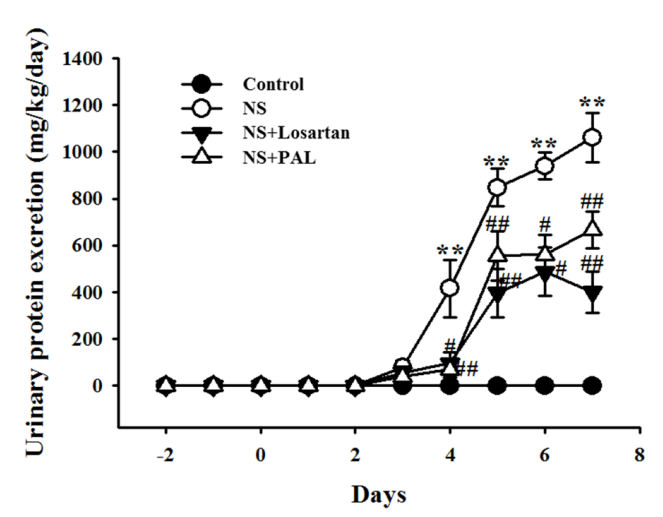

\section{B}

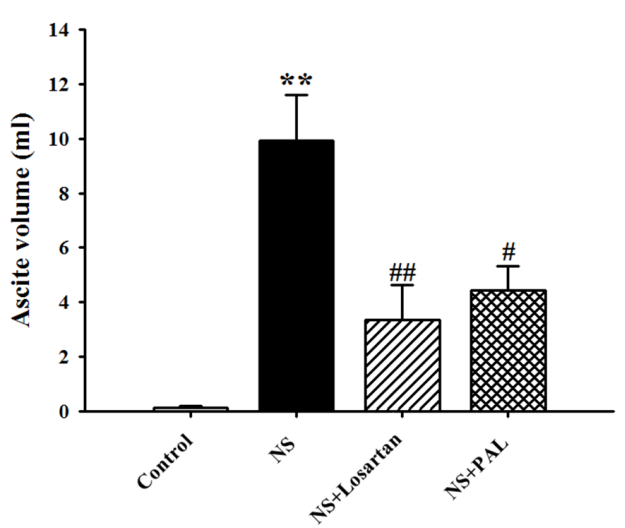

Figure 1. Effects of Plantago asiatica L. (PAL) on urinary protein excretion at various time points (A) and ascites $(\mathbf{B})$. Values were expressed as mean $\pm \mathrm{SE}(n=7) .{ }^{* *} p<0.01$ versus Control; ${ }^{\#} p<0.05,{ }^{\# \#} p<0.01$ versus NS.

\subsection{Effect of PAL on Plasma Parameters and Renal Function}

The NS group exhibited increases in T-Cho, TG and LDL-c levels, which are clinical data of nephrotic syndrome. However, the PAL-treated group exhibited significantly lower levels of these markers (Table $1, p<0.05$ ). Treatment with losartan yielded similar results to those of the PAL-treated group. As indicated in Table 2, compared with the Control group, the NS group exhibited increased plasma BUN and creatinine levels. However, results from the PAL-treated group demonstrated attenuation of these increased levels $(p<0.01)$. In addition, intravenous puromycin aminonucleoside decreased T-pro and Alb levels. However, the PAL-treated group had significantly increased T-pro and Alb levels $(p<0.05)$. Again, treatment with losartan yielded similar results. These results indicated that PAL could improve renal function and reduce hyperlipidemia in puromycin aminonucleoside-induced NS rats.

Table 1. Effect of treatment PAL on plasma lipids.

\begin{tabular}{ccccc}
\hline \multirow{2}{*}{ Groups } & \multirow{2}{*}{ Control } & NS & \multicolumn{2}{c}{ NS } \\
\cline { 4 - 5 } & & & Losartan & PAL \\
\hline T-Cho $(\mathrm{mg} / \mathrm{dL})$ & $63.4 \pm 3.2$ & $397.8 \pm 2.3^{* *}$ & $258.2 \pm 38.4^{\#}$ & $301.8 \pm 34.0^{\#}$ \\
TG $(\mathrm{mg} / \mathrm{dL})$ & $97.6 \pm 16.1$ & $196.6 \pm 3.4^{* *}$ & $352.3 \pm 65.3^{\#}$ & $355.2 \pm 41.1^{\# \#}$ \\
LDL-c $(\mathrm{mg} / \mathrm{dL})$ & $20.8 \pm 3.6$ & $171.2 \pm 9.0^{* *}$ & $90.6 \pm 20.9^{\# \#}$ & $110.6 \pm 22.0^{\#}$ \\
\hline
\end{tabular}

Values were expressed as mean $\pm \mathrm{SE}(n=7) .{ }^{* *} p<0.01$ versus Control; ${ }^{\#} p<0.05,{ }^{\# \#} p<0.01$ versus NS. Abbreviations: T-Cho, total cholesterol; TG, triglyceride; LDL-c, low-density lipoprotein cholesterol. 
Table 2. Effect of treatment PAL on renal functional parameters.

\begin{tabular}{|c|c|c|c|c|}
\hline \multirow{2}{*}{ Groups } & \multirow{2}{*}{ Control } & \multirow{2}{*}{ NS } & \multicolumn{2}{|c|}{ NS } \\
\hline & & & Losartan & PAL \\
\hline BUN (mg/dL) & $13.7 \pm 0.8$ & $56.4 \pm 5.5^{* *}$ & $33.9 \pm 6.3^{\#}$ & $30.8 \pm 3.6$ \#\# \\
\hline T-pro (mg/dL) & $5.6 \pm 0.1$ & $3.6 \pm 0.1^{* *}$ & $4.8 \pm 0.3^{\# \#}$ & $4.2 \pm 0.2^{\#}$ \\
\hline $\mathrm{Alb}(\mathrm{mg} / \mathrm{dL})$ & $4.1 \pm 0.1$ & $1.1 \pm 0.2 * *$ & $2.9 \pm 0.4^{\# \#}$ & $2.2 \pm 0.3^{\# \#}$ \\
\hline Plasma creatinine $(\mathrm{mg} / \mathrm{dL})$ & $0.11 \pm 0.01$ & $0.25 \pm 0.03^{* *}$ & $0.13 \pm 0.022^{\# \#}$ & $0.15 \pm 0.01$ \\
\hline
\end{tabular}

Values were expressed as mean $\pm \operatorname{SE}(n=7)$. ${ }^{* *} p<0.01$ versus Cont.; ${ }^{\#} p<0.05,{ }^{\# \#} p<0.01$ versus NS. Abbreviations: BUN, blood urea nitrogen; T-pro, total protein; Alb, albumin.

\subsection{Effect of PAL on Renal Morphology}

There were no histopathological changes in the glomerular structures in any of the groups (Figure 2). In parallel with the proteinuria, the puromycin aminonucleoside-treated rats exhibited marked pathological lesions characterized by abundant protein exudation in renal tubular lumens and marked interstitial inflammatory cell infiltration. However, treatment with PAL attenuated the pathological damage to some degree, by reducing inflammatory cell infiltration and protein cast formation. In addition, pathological scores were significantly decreased. Treatment with losartan yielded similar results to PAL. These results implied that PAL could alleviate pathological damage to the kidney in puromycin aminonucleoside-induced NS rats.
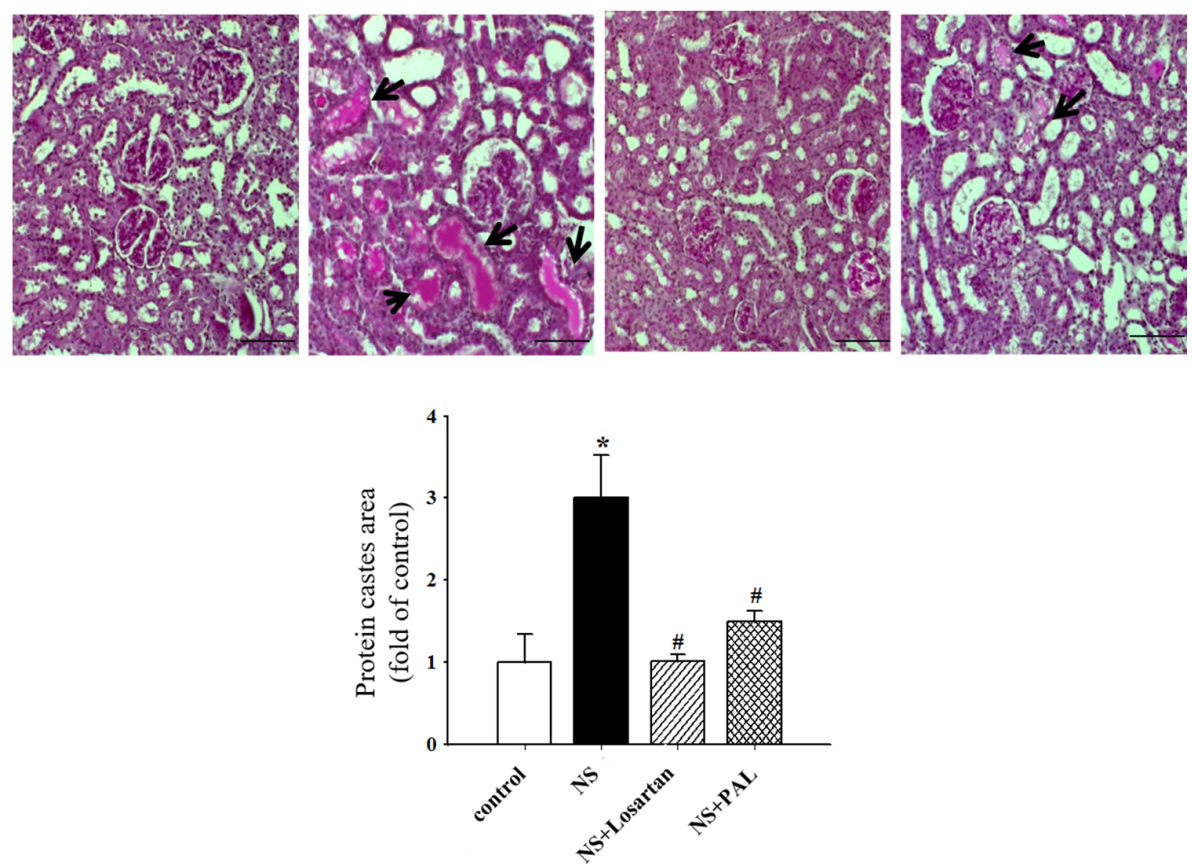

Figure 2. Effects of treatment of PAL on renal morphology. Representative photomicrographs of PAS (Periodic acid-chiff)-stained tissues (magnification $\times 200$ ). The bottom panels represent quantitative assessments of protein cast area. Protein casts in the distal tubules are indicated by black arrows in the pictures. Values were expressed as mean $\pm \mathrm{SE}(n=7) .{ }^{*} p<0.05$ versus Control.; ${ }^{\#} p<0.05$ versus NS.

\subsection{Effect of PAL on Podocyte Injury}

Podocin is specifically localized to the podocyte foot process, which functions to maintain glomerular filtration permeability. The NS group exhibited significantly decreased expression of podocin in western blot and immunohistochemistry assays compared with the Control group (Figure 3). However, treatment with PAL significantly recovered the expression of podocin levels as measured by 
both techniques $(p<0.01)$. Treatment with losartan yielded similar results. These results suggest that PAL could enhance podocyte foot processes by recovering the expression of podocin in puromycin aminonucleoside-induced NS rats.

$\mathbf{A}$

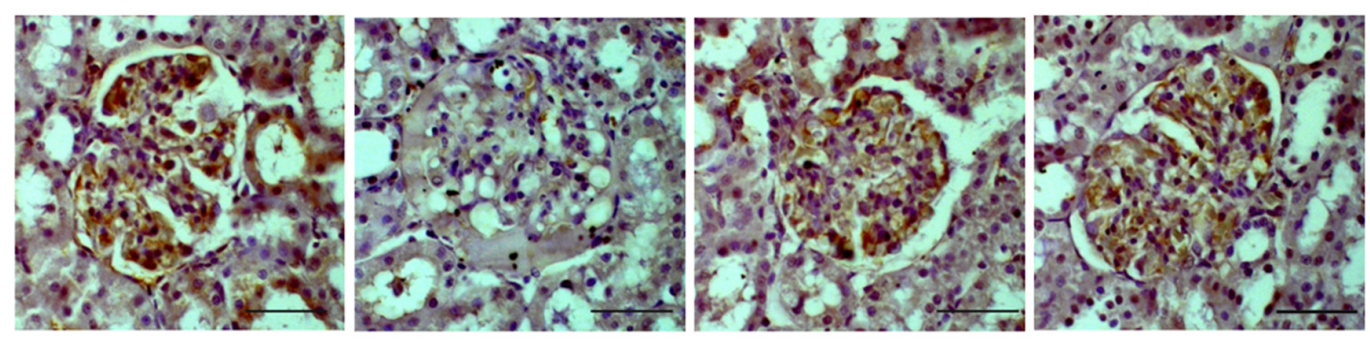

B
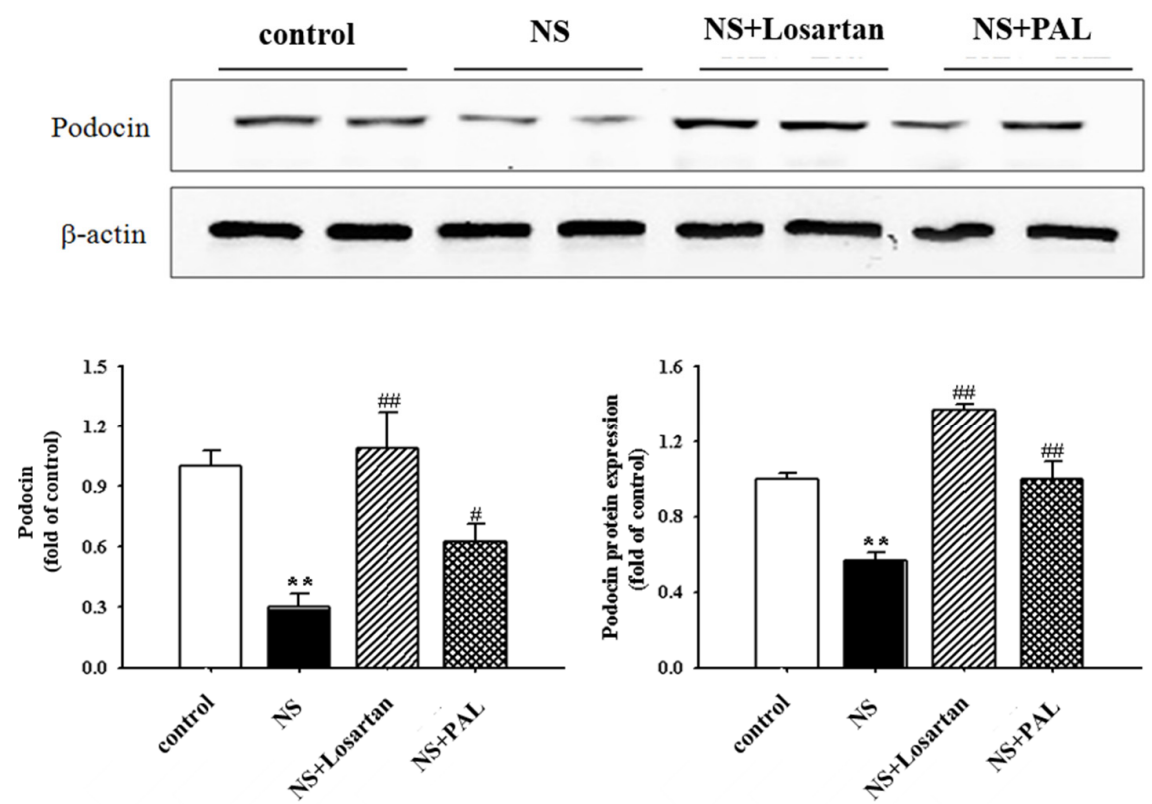

Figure 3. Effects of treatment of PAL on renal podocin expression. The top panels show immunohistochemistry staining (magnification $\times 400)(\mathbf{A})$ and western blot $(\mathbf{B})$ of renal cortical tissue. The bottom panels represent quantitative assessments of podocin expression. Values were expressed as mean $\pm \mathrm{SE}(n=7) .{ }^{* *} p<0.01$ versus Control.; ${ }^{\#} p<0.05$, ${ }^{\# \#} p<0.01$ versus NS.

\subsection{Effect of PAL on Inflammatory Markers in the Kidney}

Proteinuria induces activation of inflammatory markers in proximal tubular cells $[19,20]$. In this present study, we investigated inflammatory markers such as ICAM-1, HMGB1, MCP-1 and TNF- $\alpha$. The NS group demonstrated progressively increasing expression of these markers compared with the Control group. However, treatment with PAL significantly decreased the expression of these markers (Figure 4). Similarly, treatment with losartan resulted in decreased inflammatory markers. These results suggest that puromycin aminonucleoside-induced renal inflammation was mediated by increases in ICAM-1, HMGB1, MCP-1 and TNF- $\alpha$. Urine protein levels, therefore, correlate with inflammatory marker levels. PAL may ameliorate renal inflammation directly by reducing proteinuria. 

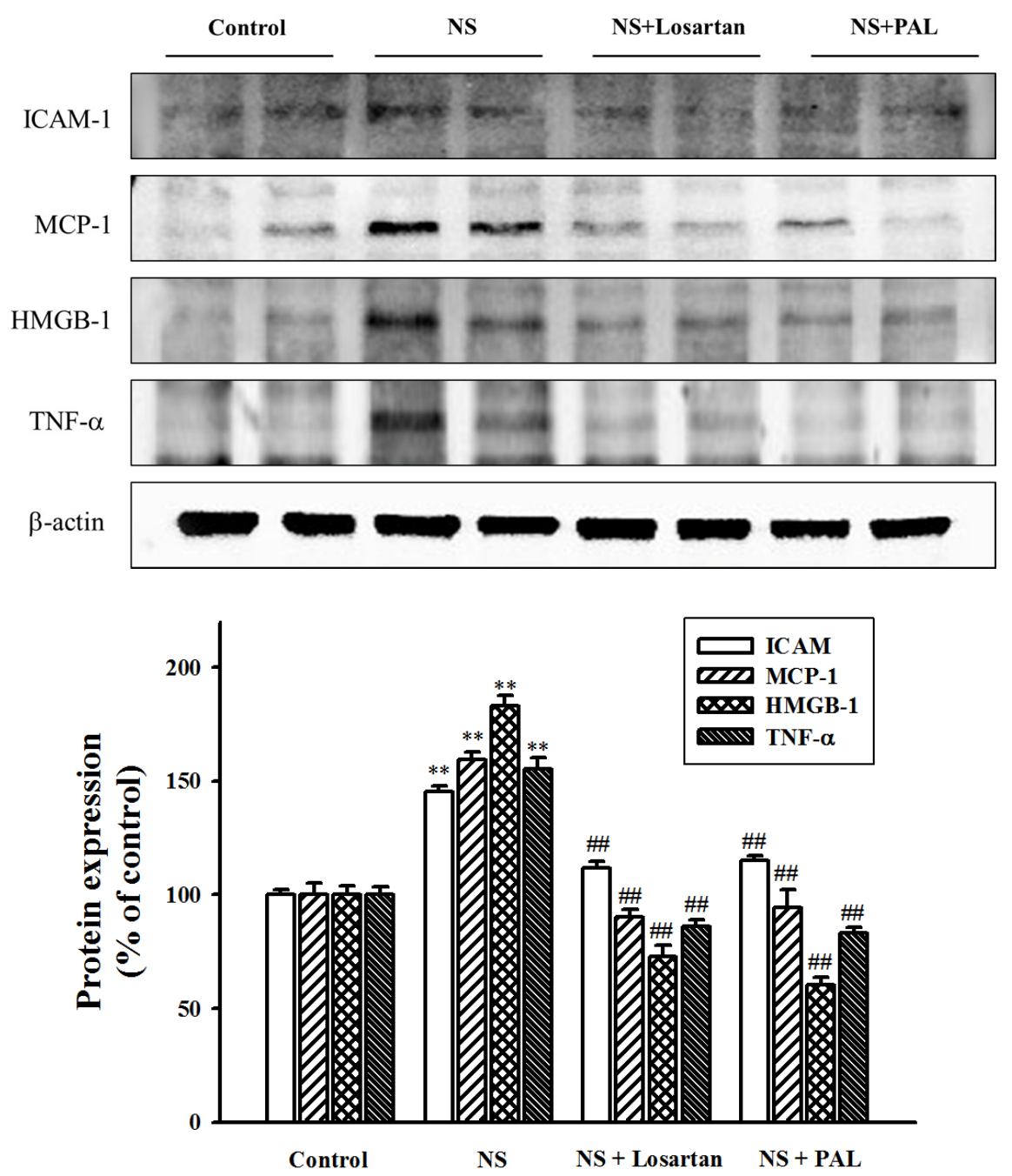

Figure 4. Effect of PAL on the expression of inflammation markers in renal tissues. The whole kidney extracts were prepared, and ICAM-1, MCP-1, HMGB-1 and TNF- $\alpha$ were analyzed by western blot analysis. Each electrophoretogram represents the results from three individual experiments. ${ }^{* *} p<0.01$ versus Control.; ${ }^{\# \#} p<0.01$ versus NS.

\subsection{Effect of PAL on Apoptosis-Related Markers in Renal Tissues}

Caspase-3, caspase-9, Bcl-2 and bax proteins direct cell apoptosis by way of intracellular and extracellular death signals. To facilitate investigation of the mode of action of PAL, concentrations of these apoptosis-related markers in the renal tissue were determined. The results showed that expression of caspase-3, caspase-9 and bax protein levels were significantly increased, whereas expression of Bcl-2 was significantly decreased in the NS group compared with the Control group. However, treatment with PAL down-regulated caspase-3, caspase-9 and bax levels, while recovering expression of Bcl-2 protein (Figure 5). Similar results were seen in the losartan-treated. These results suggest that puromycin aminonucleoside-induced renal apoptosis is due to both increases in caspase-3, caspase- 9 and bax levels, as well as reduced bcl-2 protein levels. These are direct targets for PAL in mediating the reduction of apoptosis. 

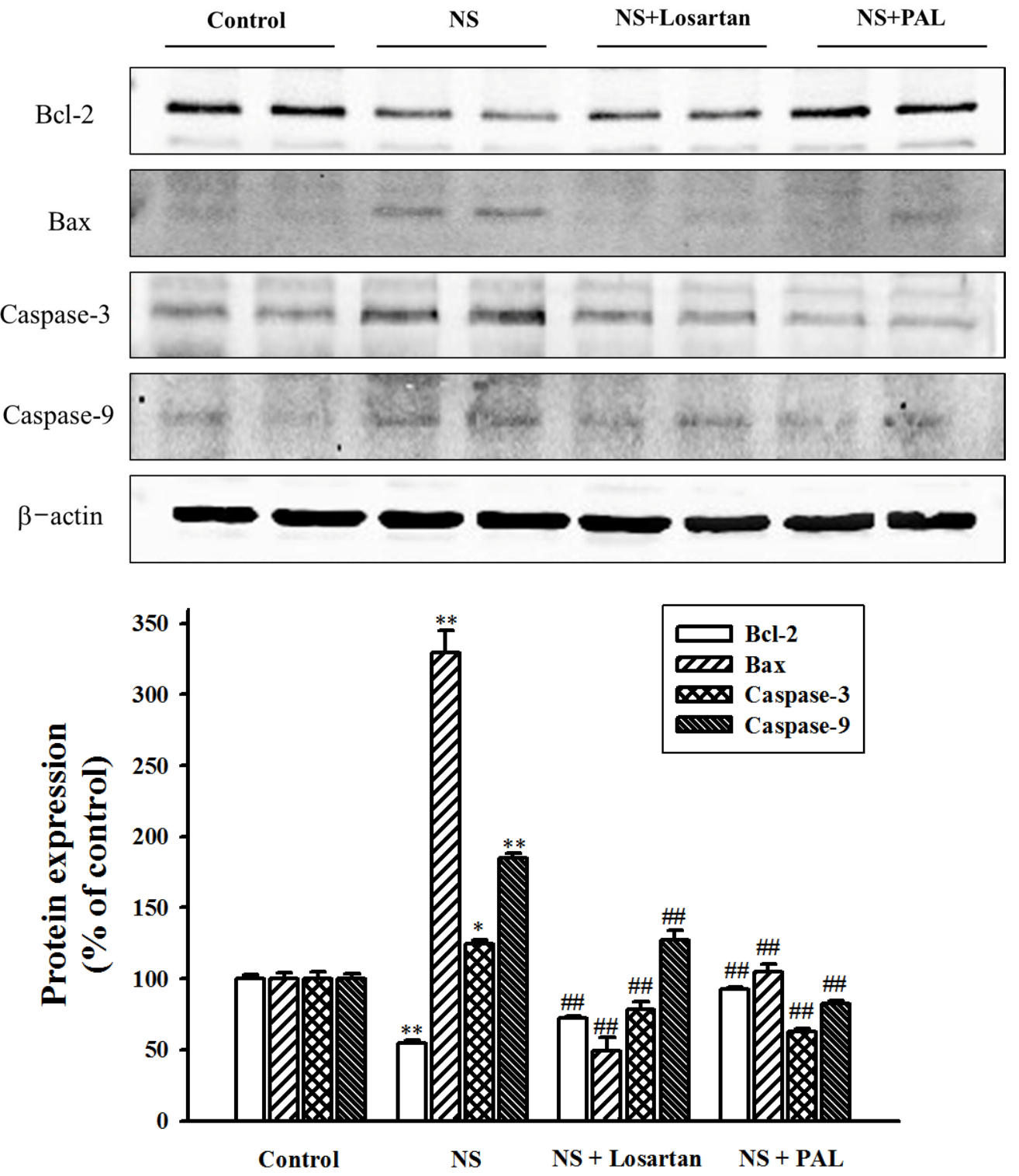

Figure 5. Effect of PAL on the expression of apoptosis-related markers in renal tissues. Whole-kidney extracts were assayed for Bcl-2, Bax, Caspase- 2 and Caspase- 9 by western blot analysis. Each electrophoretogram is representative of the results from three individual experiments. ${ }^{*} p<0.05$, ** $p<0.01$ versus Control.; ${ }^{\# \#} p<0.01$ versus NS.

\subsection{Effect of PAL on MAPK Signal Pathway in Renal Tissues}

Activation of MAP kinases, such as ERK, JNK and p38, is associated with renal injury involving cellular changes such as cell proliferation, inflammation, and apoptosis in renal cells [21]. To determine whether MAP kinases are involved in the progression of puromycin aminonucleoside-induced nephrotic syndrome, we examined the phosphorylated form of each MAP kinase. Our results showed that the puromycin aminonucleoside-injected group exhibited increased levels of the phosphorylated forms of ERK, JNK, and p38. However, treatment with PAL suppressed these increases (Figure 6). Treatment with losartan yielded similar results. In addition, the results demonstrated no increase in total ERK, JNK and p38 levels in any group. 

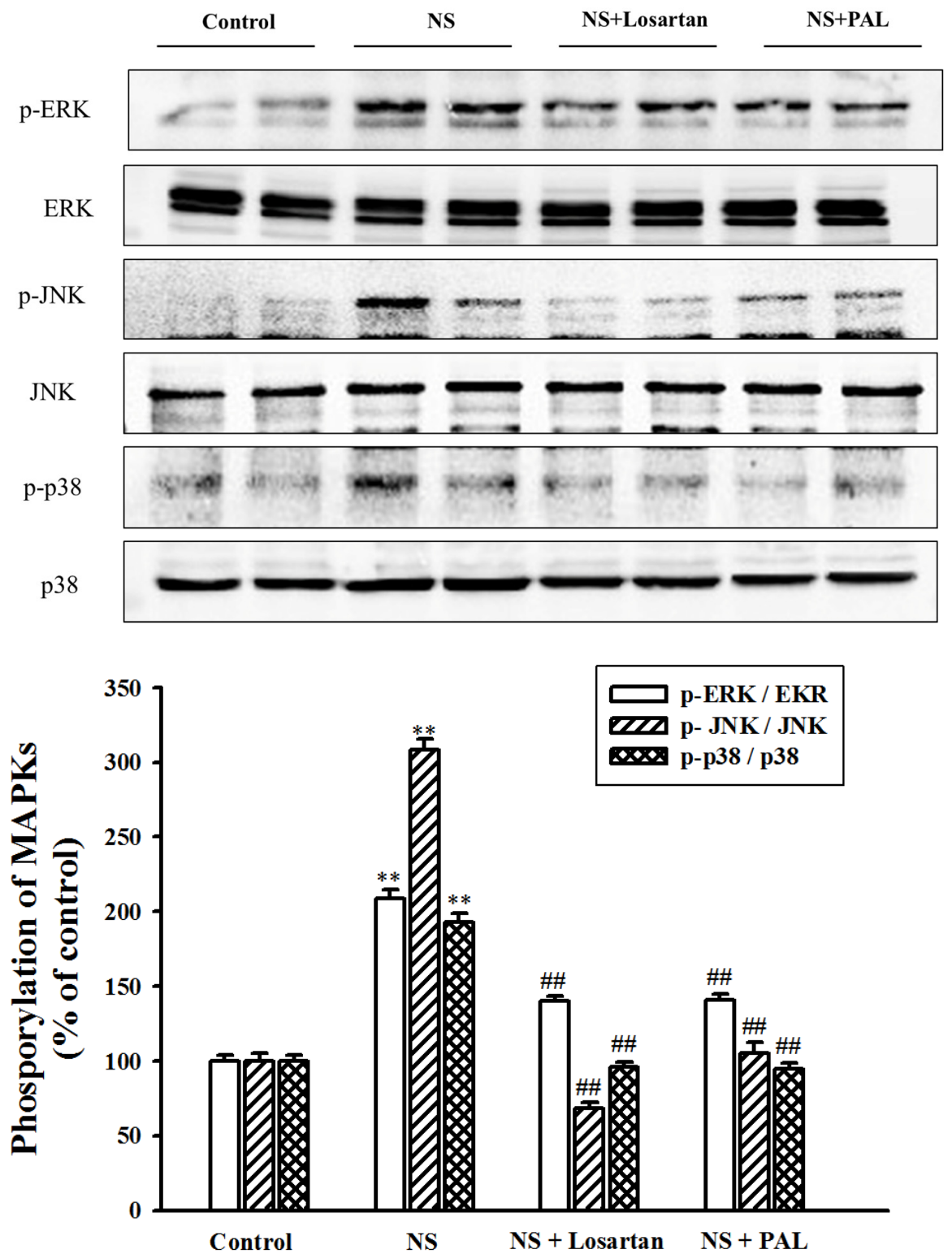

Figure 6. Effect of PAL on the expression of MAPK in renal tissues. MAPKs were detected by specific antibodies and compared to the corresponding signals from phosphorylated MAPKs. Whole-kidney extracts were assayed for phosphorylated MAPKs western blot analysis. Each electrophoretogram is representative of the results from three individual experiments. ${ }^{* *} p<0.01$ versus Control.; ${ }^{\# \#} p<0.01$ versus NS.

\section{Discussion}

Nephrotic syndrome (NS) is one of the most common glomerular diseases, which include proteinuria, hypoalbuminemia, hyperlipidemia and edema [3]. Despite the advanced technology and drugs that have been or are currently being developed to treatment and treat nephrotic syndrome, existing pharmaceutical treatments are not sufficient to treat NS patients from developing renal diseases [22]. Therefore, it is necessary to seek out other effective drugs to target and treat NS.

The present study used a single puromycin aminonucleoside injection method to induce a model NS, the features of which are similar to those of the clinical syndrome. This model has been widely used in previous studies [22-24]. Typically, this model results in histological abnormalities of glomerular epithelial and endothelial cells. Many previous studies have demonstrated that proteinuria 
is accompanied by kidney lesions. Increased inflammation and apoptosis may be the underlying mechanism of these changes [7-9].

Maintenance of glomerular filtration by podocytes depends on podocin, nephrin and CD2-associated proteins, which are known to be involved in maintaining the structural integrity of the slit diaphragms [25]. Thus, podocyte apoptosis is an important factor in puromycin aminonucleoside-induced nephrosis and the progression of focal segmental glomerulosclerosis [26]. Results of the present study, showed that treatment with PAL significantly decreased 24-h proteinuria and edema in puromycin aminonucleoside-induced nephrosis. In addition, the increased podocin expression, higher plasma levels of albumin and protein and decrease in protein cast formation in our morphological study were correlated with the inhibition of proteinuria. These results provide evidence that PAL may represent a novel therapy for NS.

As inflammation and apoptosis promote progression of renal injuries in a puromycin aminonucleoside-induced NS model, we hypothesize that the renoprotective function of PAL may be partly due to its anti-inflammatory and anti-apoptosis effects. Intracellular adhesion molecules (ICAM-1), monocyte chemotactic protein-1 (MCP-1), tumor necrosis factor alpha (TNF- $\alpha$ ) and high-mobility group box-1 (HMGB1) are pro-inflammatory cytokines, chemokines and/or proteins. They are strongly associated with the activation and progression of renal injury and inflammation, as well as triggering cell death in various kidney cells, including tubular epithelial cells, podocytes, mesangial cells and endothelial cells [27]. Several studies have reported that increases in these factors are associated with increased proteinuria and leakage of albumin and proteins in patients with renal diseases $[19,20]$. The present study demonstrated that PAL ameliorated increases in pro-inflammatory cytokines, chemokines and proteins. This suggests that PAL may inhibit puromycin aminonucleoside-induced nephrosis.

Hyperlipidemia is also one of the main features of puromycin aminonucleoside-induced NS in rats [22-24]. Several reports have suggested that apoptosis of the tubular epithelium is probably attributable to the impact of proteinuria and lipid toxicity. In addition, albumin bound to fatty acids in urine is reabsorbed by proximal tubular epithelial cells and causes apoptosis and renal damage [28,29]. The present study showed that treatment with PAL significantly decreased plasma levels of T-Cho, TG, and LDL-c. Therefore, our study deduced that the reduction of apoptosis by treatment with PAL may be partially due to its down-regulation and moderation of lipid levels, thus decreasing urinary protein levels.

To clarify the anti-apoptotic mechanism of PAL, our study investigated the influence of PAL on the expression of major apoptosis regulating factors such as caspase-3, caspase-9, Bcl-2 and bax. Caspases and bax are major apoptosis effectors, being considered the protein triggers for cell death. Proteins of the Bcl family, such as Bcl-2, play a pivotal anti-apoptotic role, maintaining cellular homeostasis through suppression of the endoplasmic reticulum. In addition, they have the ability to inhibit pro-apoptotic mechanisms and promote cell survival $[30,31]$. The present study showed that PAL could down-regulate apoptotic factors such as caspase-3, caspase-9 and bax, as well as up-regulating anti-apoptotic factors such as Bcl-2. In this way, PAL may act as an effective anti-apoptotic agent.

Renal function is closely correlated with urinary protein excretion level and is a direct reflection of progression of renal disease. The concentrations of plasma BUN and creatinine, which are important indices reflecting renal function, depend on the glomerular filtration rate (GFR). Renal dysfunction reduces their filtration, and therefore levels rise [32]. In the present study, our results showed that levels of plasma creatinine and blood urea nitrogen were increased and levels of plasma albumin were decreased by injection of puromycin aminonucleoside, indicating that renal filtration function was impaired by puromycin aminonucleoside. However, treatment with PAL counteracted these changes, indicating that PAL could enhance renal filtration in puromycin aminonucleoside-induced NS rats.

The mitogen-activated protein kinases (MAPKs) react to extracellular stimuli (mitogens) and control various cellular activities including gene expression, mitosis, differentiation, cell survival and apoptosis [33] MAP kinases regulate important cellular functions through the activation of specific 
signal transduction pathways from the cell surface to the nucleus [34]. It has been reported that these kinases are activated by numerous factors implicated in the pathogenesis of various renal injuries, including cytokines and cellular stress [35]. In addition, MAPKs also control cellular responses to cytokines and stress, both of which are related to inflammation and the immune system. In addition, they play a critical role in modulating the NF-kB pathway [36,37]. Recently, several studies have suggested that the MAP kinase family is activated in the kidney, contributing to progressive renal injury in, for example, ischemia-reperfusion, modeled diabetes and puromycin aminonucleoside-induced nephropathy [21,38]. In the present study, the results showed that PAL decreased the expression of MAP kinases such as ERK, JNK and p38. Therefore, our study demonstrated that the regulating of MAP kinase by treatment with PAL may inhibit signaling of inflammation and apoptosis in renal injury [39]. A major circulating factor in FSGS is soluble urokinase receptor suPAR, which has also a role as inflammatory mediator [40]. This molecule was not analyzed in our study but future investigations will address this.

There are some limitations from not providing the composition of PAL and comparing glomerular ultrastructure in this study. The next study will be focused on the validation of PAL by HPLC and glomerular function in NS animal models.

\section{Conclusions}

The present study demonstrates that treatment with Plantago asiatica L. effectively reduced proteinuria, edema, concentrations of plasma creatinine and blood urea nitrogen, treated glomerular morphological change, up-regulated the expression of podocin and inhibited activation of inflammatory and apoptotic factors. Plantago asiatica L. also decreased phosphorylation of MAPKs. These results suggest that Plantago asiatica L. could be a credible new curative therapeutic option to ameliorate renal dysfunction seen in the nephrotic syndrome.

Acknowledgments: This work was supported by the National Research Foundation of Korea (NRF) grant funded by the Korean government (MSIP) (2008-0062484).

Author Contributions: M.C.K. and Y.J.L. designed the study. M.C.K., J.H.P., R.T., J.J.Y., H.Y.K. and Y.M.A. performed the experimental work and data analyses. D.G.K. and H.S.L. supervised the experimental work. All authors have read and approved the final manuscript.

Conflicts of Interest: The authors report no conflict of interest.

\section{References}

1. Orth, S.R.; Ritz, E. The nephrotic syndrome. N. Engl. J. Med. 1998, 338, 1202-1211. [CrossRef] [PubMed]

2. Eckardt, K.U.; Coresh, J.; Devuyst, O.; Johnson, R.J.; Köttgen, A.; Levey, A.S.; Levin, A. Evolving importance of kidney disease from subspecialty to global health burden. Lancet 2013, 382, 158-169. [CrossRef]

3. Bulucu, F.; Vural, A.; Aydin, A.; Sayal, A. Oxidative stress status in adults with nephrotic syndrome. Clin. Nephrol. 2000, 53, 169-173. [PubMed]

4. Camici, M. The nephrotic syndrome is an immune inflammatory disorder. Med. Hypotheses 2007, 68, 900-905. [CrossRef] [PubMed]

5. Imig, J.D.; Ryan, M.J. Immune and inflammatory role in renal disease. Compr. Physiol. 2013, 3, 957-976. [PubMed]

6. Sakurai, H.; Hisada, Y.; Ueno, M.; Sugiura, M.; Kawashima, K.; Sugita, T. Activation of transcription factor NF-kappa B in experimental glomerulonephritis in rats. Biochim. Biophys. Acta 1996, 1316, 132-138. [CrossRef]

7. Wang, Y.; Harris, D.C. Macrophages in renal disease. J. Am. Soc. Nephrol. 2011, 22, 21-27. [CrossRef] [PubMed]

8. Sanz, A.B.; Santamaria, B.; Ruiz-Ortega, M.; Edido, J.; Ortiz, A. Mechanism of renal apoptosis in health and disease. J. Am. Soc. Nephrol. 2008, 19, 1634-1642. [CrossRef] [PubMed] 
9. Zoja, C.; Abbate, M.; Remuzzi, G. Progression of renal injury toward interstitial inflammation and glomerular sclerosis is dependent on abnormal protein filtration. Nephrol. Dial. Transplant. 2015, 30, 706-712. [CrossRef] [PubMed]

10. Hsiago, W.L.; Liu, L. The role of traditional Chinese herbal medicines in cancer therapy from TCM theory to mechanistic insights. Planta Med. 2010, 76, 1118-1131. [CrossRef] [PubMed]

11. Ito, T.; Seo, N.; Yagi, H.; Ohtani, T.; Tokura, Y.; Takigawa, M.; Furukawa, F. Unique therapeutic effects of the Japanese-Chinese herbal medicine, Saireito, on Th1/Th2 cytokines balance of the autoimmunity of MRL/lpr mice. J. Dermatol. Sci. 2002, 28, 198-210. [CrossRef]

12. Wang, W.; Wang, S. Nephrotic syndrome and the TCM treatment. J. Tradit. Chin. Med. 2004, 24, $201-203$. [PubMed]

13. Wei, L.; Ye, R.; Luan, T.; Lu, R.; Chen, B. Long term effect of TCM decoctions in treatment of nephrotic syndrome. J. Tradit. Chin. Med. 2002, 22, 83-86. [PubMed]

14. Samuelsen, A.B. The traditional uses, chemical constituents and biological activities of Plantago major L. A review. J. Ethnopharmacol. 2000, 71, 1-21. [CrossRef]

15. Xu, C.; Luo, L.; Tan, R.X. Antidepressant effect of three traditional Chinese medicines in the learned helplessness model. J. Ethnopharmacol. 2004, 91, 345-349. [CrossRef] [PubMed]

16. Hannan, J.M.A.; Ali, L.; Khaleque, J.; Akhter, M.; Flatt, P.R.; Abdel-wahab, Y.H.A. Aqueous extracts of husks of Plantagoovata reduce hyperglycemia in type 1 and type 2 diabetes by inhibition of intestinal glucose absorption. Br. J. Nutr. 2006, 96, 131-137. [CrossRef] [PubMed]

17. Yin, J.Y.; Nie, S.P.; Zhou, C.; Wan, Y.; Xie, M.Y. Chemical characteristics and antioxidant activities of polysaccharide purified from the seeds of Plantago asiatica L. J. Sci. Food Agric. 2010, 90, 210-217. [CrossRef] [PubMed]

18. Nandi, D.; Patra, R.C.; Swarup, D. Effect of cysteine, methionine, ascorbic acid and thiamine on arsenic-induced oxidative stress and biochemical alterations in rats. Toxicology 2005, 211, 26-35. [CrossRef] [PubMed]

19. Koshikawa, M.; Mukoyama, M.; Mori, K.; Suganami, T.; Sawai, K.; Yoshioka, T. Role of p38 mitogen-activated protein kinase activation in podocyte injury and proteinuria in experimental nephrotic syndrome. J. Am. Soc. Nephrol. 2005, 16, 2690-2701. [CrossRef] [PubMed]

20. Gomez-chiari, M.; Ortiz, A.; Lerma, J.L.; Lopez-armada, M.J.; Mampaso, F.; Gonzalez, E. Involvement of tumor necrosis factor and platelet-activating factor in the pathogenesis of experimental nephrosis in rats. Lab. Investig. 1994, 70, 449-459.

21. Ma, F.Y.; Liu, J.; Nikolic-paterson, D.J. The role of stress-activated protein kinase signaling in renal pathophysiology. Braz. J. Med. Biol. Res. 2009, 42, 29-37. [CrossRef] [PubMed]

22. Reiser, J.; Sever, J. Podocyte biology and pathogenesis of kidney disease. Annu. Rev. Med. 2013, 64, $357-366$. [CrossRef] [PubMed]

23. Fukuda, H.; Hidaka, T.; Takagi-akiba, M.; Trejo, J.A.O.; Sasaki, Y.; Wang, J.; Asanuma, K.; Tomino, Y. Podocin is translocated to cytoplasm in puromycinaminonucleoside nephrosis rats and in poor-prognosis patients with IgA nephropathy. Cell Tissue Res. 2015, 360, 391-400. [CrossRef] [PubMed]

24. Takeuchi, S.; Hiromura, K.; Tomioka, M.; Takahashi, S.; Maeshima, A.; Kaneko, Y.; Kuroiwa, T.; Nojiam, Y. The immunosuppressive drug mizoribine directly prevents podocyte injury in puromycinaminonucleoside nephrosis. Nephron Exp. Nephrol. 2010, 116, 3-10. [CrossRef] [PubMed]

25. Bohr, D.C.; Koch, M.; Kritzenberger, M.; Fuchshofer, R.; Tamm, E.R. Increase expression of olfactomedin-1 and myocilin in podocytes during puromycinaminonucleoside nephrosis. Nephrol. Dial. Transplant. 2011, 26, 83-92. [CrossRef] [PubMed]

26. Coward, R.J.; Foster, R.R.; Patton, D.; Ni, L.; Lennon, R.; Bates, D.O.; Harper, S.J.; Mathieson, P.W.; Saleem, M.A. Nephrotic plasma alters slit diaphragm dependent signaling and translocatesnephrin, podocin and CD2 associated protein in cultured human podocytes. J. Am. Soc. Nephrol. 2015, 16, 629-637. [CrossRef] [PubMed]

27. Fernandez, L.; Romero, M.; Soto, H.; Mosquera, J. Increase apoptosis in acute puromycinaminonucleoside nephrosis. Exp. Nephrol. 2001, 9, 99-108. [CrossRef] [PubMed]

28. Lau, A.; Wang, S.; Liu, W.; Haig, A.; Zhang, Z.X.; Jevnikar, A.M. Glycyrrhizic acid ameliorates HMGB1-mediated cell death and inflammation after renal ischemia reperfusion injury. Am. J. Nephrol. 2014, 40, 84-95. [CrossRef] [PubMed] 
29. Ohse, T.; Inagi, R.; Tanaka, T.; Ota, T.; Miyata, T.; Kojima, I. Albumin induces endoplasmic reticulum stress and apoptosis in renal proximal tubular cells. Kidney Int. 2006, 70, 1447-1455. [CrossRef] [PubMed]

30. Lee, H.S. Mechanisms and consequences of hypertriglyceridemia and cellular lipid accumulation in chronic kidney disease and metabolic syndrome. Histol. Histopathol. 2011, 26, 1599-1610. [PubMed]

31. Soriano, M.E.; Scorrano, L. The interplay between BCL-2family proteins and mitochondrial morphology in the regulation of apoptosis. Adv. Exp. Med. Biol. 2010, 687, 97-114. [PubMed]

32. Riedl, S.J.; Shi, Y. Molecular mechanisms of caspase regulating during apoptosis. Nat. Rev. Mol. Cell Biol. 2004, 5, 897-907. [CrossRef] [PubMed]

33. Pearson, G.; Robinson, F.; Beers Gibson, T.; Xu, B.; Karandikar, M.; Berman, K.; Cobb, M. Mitogen-activated protein (MAP) kinase pathways: Regulation and physiological functions. Endocr. Rev. 2001, 22, 153-183. [PubMed]

34. Singh, K.; Ray, R.; Sharma, A.; Gupta, R.; Bagga, A.; Dinda, A.K. Peritubular capillaries and renal function in pediatric idiopathic nephrotic syndrome. Saudi J. Kidney Dis. Transplant. 2013, 24, 942-949.

35. Chang, L.; Karin, M. Mammalian MAP kinase signaling cascades. Nature 2001, 410, 37-40. [CrossRef] [PubMed]

36. Surh, Y.; Chun, K.; Cha, H.; Han, S.; Keum, Y.; Park, K.; Lee, S. Molecular mechanisms underlying chemopreventive activities of anti-inflammatory phytochemicals: Down-regulation of COX-2 and iNOS through suppression of NF-kappa B activation. Mutat. Res. 2001, 480, 243-268. [CrossRef]

37. Wu, H.; Zhao, G.; Jiang, K.; Chen, X.; Zhu, Z.; Qiu, C.; Li, C.; Deng, G. Plantamajoside ameliorates lipopolysaccharide-induced acute lung injury via suppressing NF- $\mathrm{kB}$ and MAPK activation. Int. Immunopharmacol. 2016, 351, 315-322. [CrossRef] [PubMed]

38. Morooka, H.; Bonventre, J.V.; Pombo, M.C.; Kyriakis, J.M.; Force, T. Ischemia and reperfusion enhance ATF-2 and c-Jun binding to CAMP response elements and to an AP-1 binding site from the c-Jun promoter. J. Biol. Chem. 1995, 270, 30084-30092. [PubMed]

39. Park, S.J.; Jeong, K.S. Cell-type-specific activation of mitogen-activated protein kinasesin PAN-induced progressive renal disease in rats. Biochem. Biophys. Res. Commun. 2004, 323, 1-8. [CrossRef] [PubMed]

40. Hahm, E.; Wei, C.; Fernandez, I.; Li, J.; Tardi, N.J.; Tracy, M.; Wadhwani, S.; Cao, Y.; Peev, V.; Zloza, A.; et al. Bone marrow-derived immature myeloid cells are a main source of circulating suPAR contributing to proteinuric kidney disease. Nat. Med. 2017, 23, 100-106. [CrossRef] [PubMed]

(C) 2017 by the authors. Licensee MDPI, Basel, Switzerland. This article is an open access article distributed under the terms and conditions of the Creative Commons Attribution (CC BY) license (http:/ / creativecommons.org/licenses/by/4.0/). 